\title{
On Energy Efficiency of Prioritized IoT Systems
}

\author{
${ }^{\dagger}$ Abdulrahman Alabbasi, ${ }^{\ddagger}$ Basem Shihada, and ${ }^{\dagger}$ Cicek Cavdar \\ $\dagger$ KTH Royal Institute of Technology, Email: \{alabbasi, cavdar\}@kth.se \\ ${ }^{\ddagger}$ King Abdullah University of Science and Technology (KAUST), Email: basem.shihada@kaust.edu.sa
}

\begin{abstract}
The inevitable deployment of 5G and the Internetof-Things (IoT) sheds the light on the importance of the energy efficiency (EE) performance of Device-to-Device (DD) communication systems. In this work, we address a potential IoT application, where different prioritized DD system, i.e., Low-Priority (LP) and High-Priority (HP) systems, co-exist and share the spectrum. We maximize the EE of each system by proposing two schemes. The first scheme optimizes the individual transmission power and the spatial density of each system. The second scheme optimizes the transmission power ratio of both systems and the spatial density of each one. We also construct and analytically solve a multi-objective optimization problem that combines and jointly maximizes both HP and LP EE performance. Unique structures of the addressed problems are verified. Via numerical results we show that the system which dominates the overall EE (combined EEs of both HP and LP) is the system corresponding to the lowest power for low/high power ratio (between HP and LP systems). However, if the power ratio is close to one, the dominating $\mathrm{EE}$ corresponds to the system with higher weight.
\end{abstract}

Index Terms-Spectrum sharing, Energy efficiency, Resource allocation, Spatial Randomness, Device-to-Device,

\section{INTRODUCTION}

High throughput data communication and reliable (long lifetime and large capacity) sensor, DD, and cellular networks are essential for the realization of $5 \mathrm{G}$ and Internet-of-Things (IoT). These emerging technologies result in significant power consumption requirements. Therefore, energy measurement metrics are essential for realizing future technologies and guaranteeing a certain energy efficiency (EE) performance. Researchers have been thoroughly investigating the greenness of communication networks. Variety of EE metrics have been proposed based on different factors and several communication layers [1]-[4]. To capture the spatial randomness of such networks, e.g., IoT, an interesting tool, i.e., stochastic geometry, showed promising techniques which can be utilized to provide tractable performance expressions [5].

Several works have utilized stochastic geometric tools to tackle the outage probability and spatial capacity of communication networks [6]-[10]. However, working on utilizing this tool in improving the EE is still an open research problem. The authors of [6] have tackled the EE performance of a heterogeneous network. Fairness of the sub-channel allocation has also been addressed. On the other hand, authors of [7] have addressed the EE maximization of several sharing systems from a game theory perspective. They considered noncooperative game between different systems, with and without incorporating pricing to the power control game. In a cellular system, authors of [8] have modeled a system that contains both macro basestations (MBSs) and femto-cell access points (FAPs) and their associated user equipments (UEs) as two independent Poisson point processes. They do not consider the interference between different systems, unlike our work which considers the inter/intra interference. The maximum EE is addressed, with respect to (w.r.t.) sub-channels allocation, while considering the impact of different diversity schemes. The authors of [9] modeled the two-tier cellular system's components, i.e., MBSs, small-cell access points (SAPs), and UEs as independent Poisson point process (PPP). They analyzed the data rate associated with different communication scenarios, i.e., MBS-to-UE, SAP-to-UE, and MBS-to-SAP. Also, the data rate to system's power ratio is obtained.

In this work, we consider an IoT environment where different priorities systems, operating with multi-users on the same spectrum, might co-exist. For instance, in industrial IoT, a factory might have several connected machines that contributes to the main factory operations, while other machines, such as temperature sensors etc., might have less operational priority (such as, accepting different outage priority, transmitting power, EE values). Motivated by the aforementioned IoT scenarios, we consider High-Priority system (HPS) and LowPriority system (LPS) co-exist in the same area while sharing the spectrum. We capture the spatial randomness of the nodes by utilizing the stochastic geometric approach. The targeted performance metric of all systems is the EE, defined as, the transmission capacity (successful transmissions per unit area) to power ratio. We enforce a High-Priority system (HPS)'s outage constraint on Low-Priority user (LPU) transmission to guarantee minimum HPS's quality of service (QoS).

Our contributions are summarized as follows. We maximize the individual EE of both Low-Priority system (LPS) and HPS. Maximum EE is achieved via proposing two schemes, i.e., scheme 1 and scheme 2. Optimal transmission power and spatial density of each system are derived in scheme 1 . The individual strict pseudo-concave structure of the problem is verified for scheme 1 . Whereas, Optimal transmission power ratio of both systems and spatial density of each system are derived in scheme 2. In this scheme, the joint strict pseudo-concave and quasi-concave structures of the associated problem have been verified. We then construct a combined multi-objective EE as a weighted sum of both LPU and HighPriority user (HPU) systems. In this approach, we maximize the combined EE w.r.t. LPU's and HPU's transmission power and spatial density. The global joint optimal solution set is obtained via verifying the mathematical structure of the multiobjective optimization problem and proposing an alternating algorithm, while satisfying the necessary and sufficient conditions. Numerical results showed that if the LP-to-HP power ratio is high or low $(>>1$ or $<<1)$, then the dominating 
EE is the one corresponding to the lower power. However, if LP-to-HP power ratio is close to one, then the dominating EE is the one with higher weight.

In the following, we show the distinction points between our proposed model and solutions and the existing ones. Unlike our work, the work of [6] did not derive the optimal power allocation of the associated problem. Also, the authors of [7] did not provide analytical results to both nodes' spatial density and transmission power. They also did not combine EE of several sharing systems. The problem models of [8], [9] are based on a master slave communication system, unlike our prioritized DD in-band sharing system. Also, different optimization variables and solution methodologies are considered inhere. We also do not ignore the intra/inter interference of different communication pairs, as the case of [9]. Unlike our model, the formulation of EE metric in [9], [10] does not maximizes the combination of two sharing systems' EE.

\section{System Model AND TRANSMISSION CAPACITY}

Targeting EE of spectrum sharing networks requires obtaining each system's capacity. In this section, we describe the system model and transmission capacity of each system.

\section{A. Spectrum Sharing Network Model}

In this network model, we consider two multi-user DD systems, with different priorities, that co-exist in the same area. Each pair of nodes (transmitter and receiver) belongs to either LPS or HPS network's transceivers. The set of all transmitter nodes, i.e., $\{l, h\} \in \Phi$, follows PPP distribution, where the nodes are uniformly distributed in the captured area. This distribution is well accepted in the literature because of the randomness of the nodes locations, i.e., this distribution represents the worst-case scenario among other distributions, e.g., [11]. The mean of the PPP distributed nodes is $\lambda_{q}$, where $q \in\{l, h\}$. This is interpreted as the average number of nodes in a unit area is equal to $\lambda_{q}$. Since different receiving nodes have similar received signal statistics, we therefore conduct the analysis on a receiving node at the origin of the map. The interfering nodes follow a marked Poisson point process (MPPP), i.e., transmitting nodes of a system $q$ are expressed as $\Xi_{q}=\left\{\left(\mathcal{X}_{q j}, h_{q j}\right)\right\}$. The location of the transmitter is noted as $\mathcal{X}_{q j}$ and the channel between the transmitter and the receiving node (at the origin) is $h_{q j}$. This channel follows a Rayleigh distribution with a unity mean and its modulus squared is expressed as $\left|h_{q j}\right|^{2}=\gamma_{q j}$. We also assume that all the transmission nodes in each priority system transmit with the same adaptive power, i.e., $P_{q}$ for $q \in\{l, h\}$. The received power at the desired receiver is expressed as $\left|x_{q}\right|^{-\alpha} P_{q} \gamma_{q j}$ for $q \in \Phi$, where $\left|x_{q}\right|$ is the distance to the receiving node at the origin and $\alpha$ is the pathloss exponent. Note that we omit the $j$ at the studied receiving node since it is at the origin.

\section{B. Success Probability and Transmission Capacity}

Inhere, we define the successful transmission probability of sharing network and the associated transmission capacity.

In order to express the targeted system's signal to noise and interference ratio (SINR), noted as system $t$, we consider all interferences from the same system, $t$, and the other system, $q$. The SINR of the targeted system is expressed as follows,

$$
\mathrm{SINR}_{t}=\frac{\left|x_{t}\right|^{-\alpha} P_{t} \gamma_{t i}}{\sum_{q \in \Phi} \sum_{x_{q} \in \Xi_{q}}\left|x_{q}\right|^{-\alpha} P_{q} \gamma_{q}+\mathcal{N}_{0}} .
$$

We ignore the effect of the thermal noise, since we assume the interference from all the nodes has larger effect on the desired signal compared to the thermal noise. Therefore, we consider the targeted signal to interference ratio (SIR). Hence, the successful transmission probability is defined as follows,

$$
\mathbb{P}_{t}=\operatorname{Pr}\left\{\Theta_{t} \geq \theta_{t}\right\}=\operatorname{Pr}\{\underbrace{\frac{\left|x_{t}\right|^{-\alpha} P_{t} \gamma_{t}}{\sum_{q \in \Phi} \sum_{x_{q} \in \Xi_{q}}\left|x_{q}\right|^{-\alpha} P_{q} \gamma_{q}}}_{\mathcal{I}_{t}} \geq \theta_{t}\},
$$

where $\theta_{t}$ is the desired SIR. Note that the interference is a sum of measurable functions of a MPPP. It follows that after applying several known techniques (i.e., Laplace transformation, Campbell's Theorem, random variable conditioning, and change of variable) on (2), the successful transmission probability of LPS is found as [5],

$$
\mathbb{P}_{l}=\exp \left(-\eta_{l}^{\prime}\left(\lambda_{l}+\lambda_{h}\left(P_{h} / P_{l}\right)^{2 / \alpha}\right)\right),
$$

where $\eta_{t}^{\prime}=\pi R_{t}^{2} \theta_{t}^{\frac{2}{\alpha}}$. The successful transmission probability of the HPS is found in similar lines with that in the LPS while switching the sub-script of the parameter from $x_{l}$ to $x_{h}$, where $x$ is any symbol used interchangeably for both LPS and HPS.

The transmission capacity of each system in the sharing area must be obtained to express the EE metric. The corresponding LP's transmission capacity is expressed as follows [5],

$$
\mathcal{T}_{l}=\lambda_{l} \exp \left(-\eta_{l}^{\prime}\left(\lambda_{l}+\lambda_{h}\left(P_{h} / P_{l}\right)^{2 / \alpha}\right)\right),
$$

The HP's transmission capacity is also find in similar lines with (4) while changing the sub-script because of the symmetry between both expressions.

\section{ACHIEVABLE ENERGy EFFICIENCY OF LPU AND HPU}

In this section, we formulate EE of LPU's network and derive the optimal solution, while protecting the HPU network. Following similar steps, we derive the optimal solution of EE performance of HPU network.

The EE of LPU's network is defined as the ratio of transmission capacity to power times the node density,

$$
\mathcal{E}_{l}\left(P_{l}, \lambda_{l}, P_{h}, \lambda_{h}\right)=\lambda_{l} \frac{e^{\left(-\eta_{l}^{\prime}\left(\lambda_{l}+\lambda_{h}\left(P_{h} / P_{l}\right)^{2 / \alpha}\right)\right)}}{\left(k_{t} P_{l}+k_{c}\right)},
$$

where $k_{c}$ and $k_{t}$ are assigned parameters which correspond to circuit and power amplifier constant power consumption.

The EE problem, formulated below, of LPU's system maximizes LPU's EE metric subject to maintaining a certain LPU's and HPU's outage probabilities,

$$
\begin{array}{ll}
\max & \lambda_{l} e^{\left(-\eta_{l}^{\prime}\left(\lambda_{l}+\lambda_{h}\left(P_{h} / P_{l}\right)^{2 / \alpha}\right)\right)} /\left(k_{t} P_{l}+k_{c}\right) \\
\text { s.t. } & \mathfrak{C}_{1}: 1-e^{\left(-\eta_{l}^{\prime}\left(\lambda_{l}+\lambda_{h}\left(P_{h} / P_{l}\right)^{2 / \alpha}\right)\right)} \leq \epsilon_{l} \\
& \left.\mathfrak{C}_{2}: 1-e^{\left(-\eta_{h}^{\prime}\left(\lambda_{h}+\lambda_{l}\left(P_{l} / P_{h}\right)^{2 / \alpha}\right)\right.}\right) \leq \epsilon_{h},
\end{array}
$$


where $\epsilon_{l}$ and $\epsilon_{h}$ are the outage probabilities tolerance thresholds. We propose two schemes to solve problem (6). The main difference between the two schemes is the definition of the optimization variables.

1) Scheme 1: In this scheme, we optimize LPU's transmission power, $P_{l}$, and LPU's spatial density, $\lambda_{l}$.

We begin by converting the probabilistic constraints $\mathfrak{C}_{1}$ and $\mathfrak{C}_{2}$ into an instantaneous constraint. These constraints act as boundaries for each of the optimization variables, i.e., $P_{l}$ and $\lambda_{l}$. Constraint $\mathfrak{C}_{1}$ upper bounds $P_{l}$ as follows,

$$
P_{l} \leq\left[\frac{\log \left(1-\epsilon_{h}\right)^{-1}}{\lambda_{l} \eta_{h}^{\prime}}-\frac{\lambda_{h}}{\lambda_{l}}\right]^{\frac{\alpha}{2}} P_{h}=P_{s}^{+} .
$$

Constraint $\mathfrak{C}_{2}$ lower bounds $P_{l}$ as follows,

$$
P_{l} \geq\left[\frac{\log \left(1-\epsilon_{l}\right)^{-1}}{\lambda_{h} \eta_{l}^{\prime}}-\frac{\lambda_{l}}{\lambda_{h}}\right]^{\frac{-\alpha}{2}} P_{h}=P_{s}^{-} .
$$

Problem (6) w.r.t. $P_{l}$ is difficult to solve by conventional method. That is due to the fractional, exponential, and geometrical nature of $P_{l}$ in (6a). In order to find the optimal $P_{l}$ which maximizes (6) we apply geometric optimization techniques and change of variable, on variable $P_{l}$ such that, $P_{n}=\left(P_{l}\right)^{\frac{2}{\alpha}}$ and $P_{l}=\left(P_{n}\right)^{\frac{\alpha}{2}}$. Since the new variable is a monotone function over the range of $P_{l} \geq 0$, we know from [12] that optimizing problem (6) w.r.t. $P_{n}$ is equivalent to optimizing it w.r.t. $P_{l}$. We apply transformation technique to the objective function in (6a), i.e., $\hat{\mathcal{E}}_{l}=f_{0}\left(\mathcal{E}_{l}\right)=\log \left(\mathcal{E}_{l}\right)$. It is proven in [12] that if $f_{0}($.$) is \mathbb{R} \rightarrow \mathbb{R}$ and monotonic increasing function, then maximizing $\mathcal{E}_{l}$ is equivalent to maximizing $\hat{\mathcal{E}}_{l}=f_{0}\left(\mathcal{E}_{l}\right)$. It is observed that these conditions are satisfied by the logarithmic function. Both constraints $\mathfrak{C}_{1}$ and $\mathfrak{C}_{2}$ are peak constraints on $P_{l}$, thus we apply them after obtaining the optimal $P_{l}$. Problem (6) is equivalent into the following problem,

$\max _{P_{s}^{-\frac{2}{\alpha}} \leq P_{n} \leq P_{s}^{+\frac{2}{\alpha}}} \log \left[\lambda_{l} \exp \left[-\eta_{l}^{\prime}\left[\lambda_{l}+\lambda_{h} P_{h}^{\frac{2}{\alpha}} / P_{n}\right]\right] /\left[k_{t} P_{n}^{\frac{\alpha}{2}}\right]\right]$.

The solution of (9) is summarized in the following lemma.

Lemma 1. The optimal LPU's transmission power that maximizes problem (9) is expressed as follows,

$$
\hat{P}_{l}=\min \left\{\max \left\{P_{l}^{*}, P_{s}^{-}\right\}, P_{s}^{+}\right\},
$$

where $P_{l}^{*}$ is expressed as follows,

$$
P_{l}^{*}=\left(\frac{2 \eta_{l}^{\prime} \lambda_{h}}{\alpha}\right)^{\frac{\alpha}{2}} P_{h}
$$

Proof. The proof is given in our full version work [13].

It is observed that the optimal LPU's transmission power increases with HPU transmission power and spatial density.

We now find the optimal LPU's spatial density which maximizes problem (6). We begin by transforming constraints $\mathfrak{C}_{1}$ and $\mathfrak{C}_{2}$ as upper bounds to the spatial density. Let us note the overall upper bound as follows,

$$
\lambda_{s}^{+}=\min \left(\lambda_{s 1}^{+}, \lambda_{s 2}^{+}\right),
$$

where

$$
\lambda_{s 1}^{+}=\left[\frac{\log \left(1-\epsilon_{h}\right)^{-1}}{\eta_{h}^{\prime}}-\lambda_{h}\right]\left(\frac{P_{h}}{P_{l}}\right)^{2 / \alpha},
$$

and

$$
\lambda_{s 2}^{+}=\left[\frac{\log \left(1-\epsilon_{l}\right)^{-1}}{\eta_{l}^{\prime}}-\lambda_{h}\left(\frac{P_{h}}{P_{l}}\right)^{2 / \alpha}\right] .
$$

We then rewrite problem (6) as follows,

$$
\max _{\lambda_{l} \leq \lambda_{s}^{+}} \lambda_{l} \exp \left(-\eta_{l}^{\prime}\left(\lambda_{l}+\lambda_{h}\left(P_{h} / P_{l}\right)^{2 / \alpha}\right)\right) / k_{t} P_{l} .
$$

The solution of (15) is summarized in the following lemma.

Lemma 2. The optimal LPU's spatial density that maximizes problem (15) is expressed as follows,

$$
\hat{\lambda_{l}}=\min \left\{\lambda_{s}^{+}, \frac{1}{\eta_{l}^{\prime}}\right\} .
$$

Proof. The proof is given in our full version work [13].

Note that the optimal LPU's spatial density decreases with the increase of the distance between nodes and the increase of the SIR threshold.

Note that in order to get the optimal expressions of transmission power and nodes spatial density for the HP system, we face two choices. One is to protect the LP users transmission via similar constraint to that in $\mathfrak{C}_{2}$ in (6) but from $\mathrm{HP}$ transmission perspective. In this case, both the optimal power and user density, are found in similar lines with the ones for LPS while changing the sub-script. The other choice is not to protect the LP's transmission. Hence, the upper bound on the optimal HP's transmission power is discarded. Also, one of the two upper bounds on the HPU's spatial density is discarded, as HPS does not care about protecting LPS's performance. Otherwise, all derivation steps are similar to that of LPS case.

2) Scheme 2: In this scheme, we maximize problem (6) by jointly optimizing the transmission power ratio, i.e., $\zeta_{n}=\frac{P_{h}}{P_{l}}$, and LPU's spatial density, i.e., $\lambda_{l}$. The main advantage of this scheme over scheme 1 is that the joint optimal solution is guaranteed without the necessity to use alternating algorithm. After substituting $\zeta_{s}=\left(\frac{P_{h}}{P_{l}}\right)^{\frac{2}{\alpha}}$ in problem (6), the targeted optimization problem is rewritten as follows,

$$
\begin{array}{ll}
\max _{\zeta_{s}, \lambda_{l}} & \lambda_{l} \exp \left(-\eta_{l}^{\prime}\left(\lambda_{l}+\lambda_{h} \zeta_{s}\right)\right) /\left(k_{t} P_{h} \zeta_{s}^{\frac{-\alpha}{2}}\right) \\
\text { s.t. } & \mathfrak{C}_{1}: \lambda_{l}+\lambda_{h} \zeta_{s}+\frac{1}{\eta_{l}^{\prime}} \log \left(1-\epsilon_{l}\right) \leq 0 \\
& \mathfrak{C}_{2}: \lambda_{l}+\zeta_{s}\left(\lambda_{h}+\frac{1}{\eta_{h}^{\prime}} \log \left(1-\epsilon_{h}\right)\right) \leq 0
\end{array}
$$

The solution of (17) is summarized as follows.

Theorem 1. The global joint optimal solution set of problem (17) is obtained as follows.

The optimal transmission power ratio is expressed as,

$$
\hat{\zeta_{s}}=\frac{2}{\alpha}\left[\lambda_{h}\left(\eta_{s}^{\prime}+\lambda_{1}+\lambda_{2}\right)+\frac{\lambda_{2}}{\eta_{p}^{\prime}} \log \left(1-\epsilon_{p}\right)\right]^{-1} .
$$

The optimal LPU's spatial density is expressed as,

$$
\hat{\lambda_{l}}=\left(\eta_{s}^{\prime}+\lambda_{1}+\lambda_{2}\right)^{-1}
$$


The Lagrangian multipliers $\lambda_{1}$ and $\lambda_{2}$ are obtained by solving the Karush-Kuhn-Tucker (KKT) conditions associated with $\mathfrak{C}_{1}$ and $\mathfrak{C}_{2}$, respectively.

Proof. The proof is given in our full version work [13].

Remark 1. It is interesting to note that both solutions of problem (6), under scheme 1, and problem (17), under scheme 2 , are equivalent, under some scenarios. Note that under inactive constraints, i.e., $\lambda_{1}=0$ and $\lambda_{2}=0$, the LPU's transmission power, obtained from the optimal $\zeta_{s}$ in (18), is equal to $P_{l}^{*}$ in (11). Similarly, under inactive constraints we note that $\lambda_{l}$ in (19) is equivalent to that in (16).

To maximize HPU's EE utilizing scheme 2, we use similar analogue to that of scheme 1 .

\section{LineARLy COMBINED EE LPU AND HPU Systems}

In this section, we maximize a weighted sum of $\mathcal{E}_{l}$ and $\mathcal{E}_{h}$ w.r.t. transmission power of both LPU and HPU systems in addition to the spatial density of both LPU and HPU system. This problem is extremely complicated to solve, because the joint concavity or quasi-concavity structure of the objective function w.r.t. all optimization variables cannot be verified. Therefore, we solve this problem by individually maximizing it w.r.t. each optimization variable. We then find the optimal expression for each variable. Finally, we propose an iterative algorithm, which utilizes the individual optimality of each variable to guarantee a global joint optimal solution of the problem.

The formulation of the weighted sum total EE metric problem is expressed as follows,

$$
\begin{array}{ll}
\max & \mathcal{E}_{T S}=\alpha_{l} \mathcal{E}_{l}\left(P_{l}, P_{h}, \lambda_{l}, \lambda_{h}\right)+\alpha_{h} \mathcal{E}_{h}\left(P_{l}, P_{h}, \lambda_{l}, \lambda_{h}\right) \\
\text { s.t. } & \mathfrak{C}_{1} ; \quad \mathfrak{C}_{2} ;
\end{array}
$$

where $\alpha_{s}$ and $\alpha_{p}$ are the weighting parameters of each metric. It is difficult to find a joint structure, i.e., concavity or pseudoconcavity, of problem (20) w.r.t. all optimization variables. Therefore, we begin our solution by maximizing problem (20) w.r.t. each variable separately, i.e., $P_{l}, \lambda_{l}, P_{h}$, and $\lambda_{h}$.

The optimal $P_{l}$ which maximizes problem (20) cannot be obtained in a similar way to the optimal $P_{l}$ which maximizes (6). This is due to the existence of $P_{l}$ in the exponential term of $\mathcal{E}_{h}$. Furthermore, it is not possible to, analytically, find the zeros of the first derivative of Lagrangian function to solve (20) w.r.t. $P_{l}$. That is because there is a weighted sum of exponential terms and each one includes a different function of $P_{l}$. To overcome this problem, we introduce a new variable, i.e., $P_{s p}$. This variable replaces $P_{l}$ in HPU's $\mathcal{E}_{h}$ term, i.e., $\mathcal{E}_{h}=\frac{\lambda_{h} \exp \left(-\eta_{h}^{\prime}\left(\lambda_{h}+\lambda_{l}\left(P_{s p} / P_{h}\right)^{2 / \alpha}\right)\right)}{\left(k_{t} P_{h}+k_{c}\right)}$. A new constraint must be added to link both variables, i.e., $P_{l}=P_{s p}$. We then apply the change of variables to both $P_{l}$ and $P_{s p}$, such that $P_{n}=P_{l}^{\frac{2}{\alpha}}$ and $P_{n p}=P_{s p}^{\frac{2}{\alpha}}$. The maximization problem of (20) is rewritten as follows,

$$
\begin{array}{ll}
\max _{\left(P_{s}^{-}\right)^{\frac{2}{\alpha}} \leq\left\{P_{n}, P_{n p}\right\} \leq\left(P_{s}^{+}\right)^{\frac{2}{\alpha}}} & \alpha_{l} \mathcal{E}_{l}\left(P_{n}\right)+\alpha_{h} \mathcal{E}_{h}\left(P_{n p}\right) \\
\text { s.t. } & P_{n}=P_{n p}
\end{array}
$$

Remark 2. Note that the second term of (21a) is constant w.r.t. $P_{n}$, i.e., $\frac{\alpha_{h} \lambda_{h} \exp \left(-\eta_{h}^{\prime}\left(\lambda_{h}+\lambda_{l} P_{n p} / P_{h}^{2 / \alpha}\right)\right)}{\left(k_{t} P_{h}+k_{c}\right)}$ is not a function of $P_{n}$. Whereas the first term in (21a) is constant w.r.t. $P_{n p}$, i.e., $\frac{\lambda_{l} \exp \left(-\eta_{l}^{\prime}\left(\lambda_{l}+\lambda_{h} P_{h}^{2 / \alpha} / P_{n}\right)\right)}{k_{t} P_{n}^{\alpha / 2}+k_{c}}$ is not a function of $P_{n p}$.

Utilizing Remark 2 and the geometric optimization techniques, which are used in Sec. III, we obtain the optimal value of $P_{l}$ in the following proposition.

Proposition 1. The optimal value of $P_{n}$ that maximizes (20), given $P_{h}$ and $\lambda_{h}$, is expressed as follows,

$$
\hat{P}_{n}=\min \left\{\max \left\{P_{n}^{o},\left(P_{s}^{-}\right)^{\frac{2}{\alpha}}\right\},\left(P_{s}^{+}\right)^{\frac{2}{\alpha}}\right\}
$$

where

$$
P_{n}^{o}=\left[\alpha+\sqrt{\alpha^{2}-16 \mu \eta_{s}^{\prime} \lambda_{h} P_{h}^{\frac{2}{\alpha}}} / 4 \mu\right]
$$

$P_{s}^{+}$and $P_{s}^{-}$are expressed in (7) and (8), respectively. Whereas, the optimal value of $P_{n p}$ that maximizes (20), given $P_{h}, \lambda_{l}$, and $\lambda_{h}$, is expressed as follows,

$$
\hat{P_{n p}}=\min \left\{\max \left\{P_{n p}^{o},\left(P_{s}^{-}\right)^{\frac{2}{\alpha}}\right\},\left(P_{s}^{+}\right)^{\frac{2}{\alpha}}\right\}
$$

where

$$
P_{n p}^{o}=\frac{P_{h}^{\frac{2}{\alpha}}}{\eta_{p}^{\prime} \lambda_{l}} \log \left[\frac{\eta_{p}^{\prime} \lambda_{l} \lambda_{h} \alpha_{p} e^{-\eta_{p}^{\prime} \lambda_{h}}}{\mu k_{t} P_{h}^{\frac{2}{\alpha}+1}}\right]
$$

The parameter $\mu$, in both (23) and (25), is the Lagrangian multiplier associated with the equality constraint in (21b). The value of $\mu$ is obtained by finding the zeros of the following function.

$$
g(\mu)=\left[2 w \mu-\frac{\mu P_{h}^{\frac{2}{\alpha}}}{\eta_{h}^{\prime} \lambda_{l}} \log (\mu)-\frac{\alpha}{2}\right]^{2}-\frac{\alpha^{2}}{4}+4 \mu \eta_{l}^{\prime} \lambda_{h} P_{h}^{\frac{2}{\alpha}}=0
$$

where $w=\frac{P_{h}^{\frac{2}{\alpha}}}{\eta_{p}^{\prime} \lambda_{l}}\left[\log \left(\eta_{p}^{\prime} \lambda_{l} \alpha_{h} \lambda_{h} e^{-\eta_{h}^{\prime} \lambda_{h}}\right)-\log \left(k_{t} P_{h}^{1+\frac{2}{\alpha}}\right)\right]$.

Proof. The proof is given in Appendix A.

We now solve for the value of $\lambda_{l}$ that maximizes problem (20). Utilizing similar geometric optimization techniques that are used in Sec. III and after some algebraic manipulations we find that maximizing problem (20) w.r.t. $\lambda_{l}$ is equivalent to the following maximization problem.

$$
\max _{\lambda_{l} \leq \lambda_{s}^{+}} \log \left(e^{-\eta_{l}^{\prime} \lambda_{l}}\left(a_{s} \lambda_{l}+c_{s} e^{-d_{s} \lambda_{l}}\right)\right)
$$

where $a_{s}=\frac{\alpha_{l}}{k_{t} P_{l}} \exp \left(-\eta_{l}^{\prime} \lambda_{h} \frac{P_{h}^{\frac{2}{\alpha}}}{P_{l}^{\frac{2}{\alpha}}}\right), c_{s}=\frac{\alpha_{h}}{k_{t} P_{h}} \exp \left(-\eta_{h}^{\prime} \lambda_{h}\right)$, and $d_{s}=\eta_{h}^{\prime} \frac{P_{l}^{\frac{2}{\alpha}}}{P_{h}^{\frac{2}{\alpha}}}-\eta_{s}^{\prime}$. The upper bound $\lambda_{s}^{+}$is expressed in (12). The solution of problem (27) is summarized in the following proposition.

Proposition 2. The optimal spatial density of LPU that maximizes (27), given $P_{h}, P_{l}$, and $\lambda_{h}$, is expressed as follows,

$$
\hat{\lambda}_{l}=\min \left\{\lambda_{l}^{o}, \lambda_{s}^{+}\right\}
$$


where

$$
\lambda_{l}^{o}=\left[\frac{1}{\eta_{l}^{\prime}}+\frac{1}{d_{s}} W\left[-\frac{c_{s} d_{s}\left(\eta_{l}^{\prime}+d_{s}\right)}{a_{s} \eta_{l}^{\prime}} \exp \left(-\frac{d_{s}}{\eta_{l}^{\prime}}\right)\right]\right]^{+} .
$$

Proof. To proof this proposition it is enough to show that $\mathcal{E}_{T S}$ is strictly quasi-concave with respect to $\lambda_{l}$ and has a single stationary (critical) point (details of the proof are omitted).

We optimize HPU's transmission power in similar lines as in optimizing the LPU's parameters. We apply the change of variable to $P_{h}$ such that $P_{u}=P_{h}^{\frac{2}{\alpha}}$. We then introduce a new optimization variable related to the HPU's transmission power, i.e., $P_{u s}$, and the associated equality constraint $P_{u s}=P_{u}$. The optimal value of $P_{u}$ which maximizes problem (20) is derived in the following proposition.

Proposition 3. The optimal value of $P_{u}$ that maximizes (20), given $P_{l}$ and $\lambda_{l}$, is expressed as follows,

$$
\hat{P}_{u}=\min \left\{\max \left\{P_{u}^{o},\left(P_{p}^{-}\right)^{\frac{2}{\alpha}}\right\},\left(P_{p}^{+}\right)^{\frac{2}{\alpha}}\right\}
$$

where

$$
P_{u}^{o}=\left[\frac{\alpha+\sqrt{\alpha^{2}-16 \mu_{p} \eta_{p}^{\prime} \lambda_{l} P_{l}^{\frac{2}{\alpha}}}}{4 \mu_{h}}\right]^{\frac{\alpha}{2}}
$$

and $P_{p}^{+}$and $P_{p}^{-}$are found in similar lines with (7) and (8), respectively. Whereas, the optimal value of $P_{u s}$ that maximizes (20), given $P_{l}, \lambda_{l}$, and $\lambda_{h}$, is expressed as follows,

$$
\hat{P_{u s}}=\min \left\{\max \left\{P_{u s}^{o},\left(P_{s}^{-}\right)^{\frac{2}{\alpha}}\right\},\left(P_{s}^{+}\right)^{\frac{2}{\alpha}}\right\}
$$

where

$$
P_{u s}^{o}=\frac{P_{l}^{\frac{2}{\alpha}}}{\eta_{s}^{\prime} \lambda_{h}} \log \left[\frac{\eta_{s}^{\prime} \lambda_{h} \lambda_{l} \alpha_{s} e^{-\eta_{s}^{\prime} \lambda_{l}}}{\mu_{p} k_{t} P_{l}^{\frac{2}{\alpha}+1}}\right]
$$

The parameter $\mu_{p}$ is the Lagrangian multiplier associated with the equality constraint similar to that introduced for the LPU's power optimization case, in $(21 b)$. The parameter $\mu_{h}$ is obtained by finding the zeros of the following function.

$$
g\left(\mu_{p}\right)=\left[2 w_{p} \mu_{p}-\frac{\mu_{p} P_{l}^{\frac{2}{\alpha}}}{\eta_{l}^{\prime} \lambda_{h}} \log \left(\mu_{p}\right)-\frac{\alpha}{2}\right]^{2}-\frac{\alpha^{2}}{4}+4 \mu_{p} \eta_{h}^{\prime} \lambda_{l} P_{l}^{\frac{2}{\alpha}}
$$

where $w_{p}=\frac{P_{l}^{\frac{2}{\alpha}}}{\eta_{s}^{\prime} \lambda_{h}}\left[\log \left(\eta_{s}^{\prime} \lambda_{h} \alpha_{l} \lambda_{l} e^{-\eta_{l}^{\prime} \lambda_{l}}\right)-\log \left(k_{t} P_{l}^{1+\frac{2}{\alpha}}\right)\right]$.

Proof. The proof is obtained using similar steps to the proof of Proposition 1.

In the following proposition, we find the value of $\lambda_{h}$ which maximizes problem (20).

Proposition 4. The optimal spatial density of HPU's system that maximizes (20), given $P_{h}, P_{l}$, and $\lambda_{l}$, is expressed as follows,

$$
\hat{\lambda_{h}}=\min \left\{\lambda_{h}^{o}, \lambda_{p}^{+}\right\}
$$

The parameter $\lambda_{p}^{+}$is found in similar lines with that in (12).
The expression of $\lambda_{l}^{o}$ is obtained as follows,

$$
\lambda_{h}^{o}=\left[\frac{1}{\eta_{h}^{\prime}}+\frac{1}{d_{p}} W\left[-\frac{c_{p} d_{p}\left(\eta_{h}^{\prime}+d_{p}\right)}{a_{p} \eta_{h}^{\prime}} \exp \left(-\frac{d_{p}}{\eta_{h}^{\prime}}\right)\right]\right]^{+}
$$

where $a_{p}=\frac{\alpha_{h}}{k_{t} P_{h}} \exp \left(-\eta_{h}^{\prime} \lambda_{l} \frac{P_{l}^{\frac{2}{\alpha}}}{P_{h}^{\frac{2}{\alpha}}}\right), c_{p}=\frac{\alpha_{l}}{k_{t} P_{l}} \exp \left(-\eta_{l}^{\prime} \lambda_{l}\right)$, and $d_{p}=\eta_{l}^{\prime} \frac{P_{h}^{\frac{2}{\alpha}}}{P_{l}^{\frac{2}{\alpha}}}-\eta_{p}^{\prime}$.

Proof. It is straight forward to obtain the proof following similar steps as in the proof of Proposition 2.

After finding the optimal values of $\left\{P_{n}, P_{n p}, \lambda_{l}, P_{u}\right.$, $\left.P_{u s}, \lambda_{h}\right\}$ which, individually, maximizes (20), we introduce an algorithm that enables us to jointly maximize (20). This algorithm utilizes the individual structure of the main problem in (20) w.r.t. each optimization variables, i.e., no need to verify the joint structure. The proposed algorithm, in Algorithm 1, iterates over the variables and update them in each iteration to obtain a joint optimal set. The following theorem introduces the global optimal solution of problem (20).

Theorem 2. Given the strict quasi-concave structure of problem (20) w.r.t. each of the optimization variable, the global optimal solution of problem (20) is found through two steps. First, find the optimal variables $\left(P_{l}, \lambda_{l}, P_{h}, \lambda_{h}\right)$ as in (22), (28), (30), (35), respectively. Second, update and iterate over these variables $\left(P_{l}, \lambda_{l}, P_{h}, \lambda_{h}\right)$ using the alternating algorithm, in Algorithm 1.

Proof. The proof is given in Appendix B.

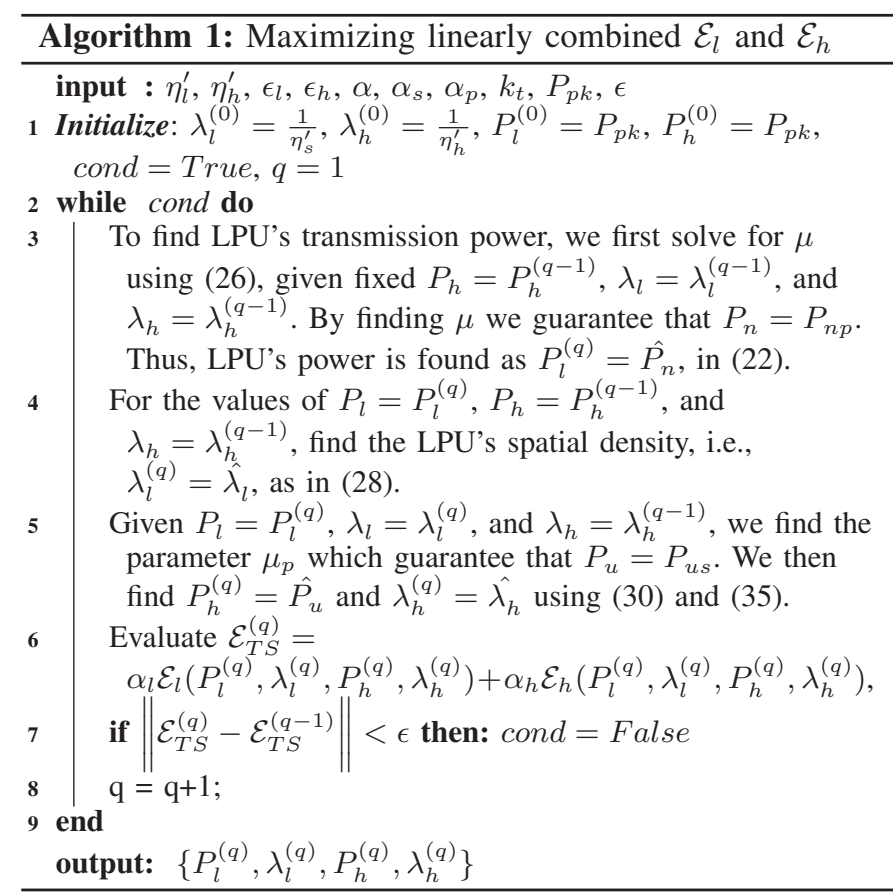

Note that $P_{p k}$, mentioned at the initialization stage of the algorithm is the maximum allowable transmission power. 


\section{Numerical Evaluation}

In this section, we evaluate the EE performance of the proposed problems, i.e., $\mathcal{E}_{l}$ (Sec. III ) and $\mathcal{E}_{T S}$ (Sec. IV). Note that through out the numerical results we use the following notations. The legend OPL is used to note that this result is associated with optimizing both spatial density and transmission power of the targeted system, whereas, OP or OL are used to note that this result is obtained by optimizing only the transmission power or the spatial density of the targeted system, respectively. The performance measure $\mathrm{EE}_{1}$ represents the LPU's EE as formulated in Sec. III, whereas, $\mathrm{EE}_{\mathrm{TS}}$ represents the linear combination of both LPU and HPU systems' EE as formulated in Sec. IV. It is worth noting that under $\mathrm{EE}_{\mathrm{TS}}$ scenario, the OPL and $\mathrm{OP}$ schemes optimize (in addition to spatial densities) w.r.t. $\zeta=\frac{P_{l}}{P_{h}}$ not $P_{l}$ and $P_{h}$, individually. Hence, the denominator of $\mathcal{E}_{l}$ and $\mathcal{E}_{h}$ in (20), becomes $P_{l}=\zeta P_{h}$ and $P_{h}$, respectively, since we assume that the HPS's power is not likely to be changed. The reason behind this substitution is that we find it more numerically stable to optimize w.r.t. to $\zeta$. The unit of $\mathrm{EE}$ is successful transmission/watt/unit-area, it could be converted to nats/joule/unit-area by multiplying the objective by $\log \left(1+\theta_{s}\right)$.

Figure 1(a) evaluates $\mathrm{EE}, \mathrm{EE}_{\mathrm{l}}$, against tolerance parameters $\epsilon_{l}$ and $\epsilon_{h}$, for different schemes (i.e., OPL, OL, and OP) and different parameter sets of $\left\{\epsilon_{l}, \epsilon_{h}\right\}$, i.e., $\{\mathrm{v}, 0.1\},\{\mathrm{v}, 0.9\}$, $\{0.9, \mathrm{v}\}$, ('v' is to note variable $\epsilon_{l}$ or $\epsilon_{h}$ ). In general, it is observed that $\mathrm{EE}_{1}$ improves with the increase of $\epsilon_{l}$ and $\epsilon_{h}$ (since the feasibility region becomes larger), except for OP with $\epsilon_{l}=0.9$ where both constraints are always inactive. Also, as expected OPL outperforms both OL and OP schemes. Under $\mathrm{OL}$ scheme, we note an increase in $\mathrm{EE}_{1}$ up-to a certain point, (a) or (b), then the performance saturates. This is because the constraint related to $\epsilon_{h}$ is active at (a), whereas, the one related to $\epsilon_{l}$ becomes inactive and the optimal $\lambda_{l}$ has been achieved at (b). Point (a) occurred before (b) because of the difference in constraint tolerance, $\epsilon_{h}=0.1,0.9$, respectively. Same behavior is noted for OP scheme, as lower power constraint is inactive after point (c), where it was active before this point. As for point (d) on scheme OPL, the node density constraint related to $\epsilon_{h}$ is active for curve $\{\mathrm{v}, 0.1\}$ (hence the optimal $\lambda_{l}$ is constant). Yet, improvement could be made for both $(\{\mathrm{v}, 0.1\}$ $\&\{\mathrm{v}, 0.9\})$ curves because of the impact of increasing $\epsilon_{l}$ on power lower bound, $P_{s}^{-}$. The saturation after point (e) on both curves $(\{\mathrm{v}, 0.1\} \&\{\mathrm{v}, 0.9\})$ occurs because there is no impact of $\epsilon_{l}$ on the optimal power. Similar behavior is inferred at (f).

Figure 1(b) evaluates the $\mathrm{EE}_{1}$ versus $\theta_{l}$ or $\theta_{h}$, noted in the legend as $\left\{\theta_{h}, \theta_{l}\right\}$ and set as $\{\mathrm{v}, 10.5\},\{\mathrm{v}, 0.5\}$, $\{10.5, \mathrm{v}\},\{0.5, \mathrm{v}\}$ ('v' is to note variable $\theta_{l}$ or $\theta_{h}$ ). It is clear that $\mathrm{EE}_{1}$ is decreasing with $\theta$, while variable $\theta_{l}$ has higher effect on the variance of $\mathrm{EE}_{1}$, in compared to variable $\theta_{h}$. Note that $\{\mathrm{v}, 10.5\}$ intersects with $\{10.5, \mathrm{v}\}$ around $\theta_{l}=10.5$ for OPL, OL, and OP curves. Hence, to have relatively high $\mathrm{EE}_{1}$ (in compared to $\mathrm{EE}_{\mathrm{h}}$ ) it is preferred to set $\theta_{l}<\theta_{h}$. As expected, it is observed that OPL scheme outperforms both OP and OL for all values of $\theta_{l}$ and $\theta_{h}$.
Figure 1(c) evaluates $\mathrm{EE}, \mathrm{EE}_{\mathrm{TS}}$, against variable $\epsilon_{l}$ and $\epsilon_{h}$, for both OL and OP schemes, under several variation of power and weight sets, i.e., $\left\{P_{l}, P_{h}, \alpha_{h}\right\}=$ $\{15,5,0.5\},\{5,15,0.5\},\{15,15,0.5\},\{0,15,0.5\},\{0,15,0.9\}$. Note that Unlike Fig. 1(a), both $\epsilon_{l}$ and $\epsilon_{h}$ change similarly, as an $\mathrm{x}$-axis. A general observation for both schemes is that if the $P_{l} / P_{h}$ ratio is high or low $(>>1$ or $<<1)$, then the dominating $\mathrm{EE}$ is the one corresponding to the lower power (if $P_{l} / P_{h}$ is low it follows that $\mathrm{EE}_{1}$ is dominating). However, if $P_{l} / P_{h}$ is close to one, then the dominating EE is the one with higher weight, $\alpha_{l}$ or $\alpha_{h}$. Also, the OP performance for different curves of $\{5,15,0.5\},\{15,15,0.5\},\{0,15,0.5\}$ is the same because we model the denominator of $\mathcal{E}_{h}$ and $\mathcal{E}_{l}$ to be $k_{t} P_{h}$ and $k_{t} P_{l}=k_{t} \zeta P_{h}$, respectively, (fixing $P_{h}$ while optimizing the ratio $\zeta$ ). Hence, the critical parameters are the constant $P_{h}$ and $\alpha_{h}$. We observe that for OL curves decreasing the ratio $P_{l} / P_{h}$ will shift the intersection point of the curves $\mathrm{OL}$ and $\mathrm{OP}$ to the right, i.e., it requires higher tolerance, $\epsilon_{l}$ and $\epsilon_{h}$, for OP to outperform OL.

\section{CONCLUSION}

In this work, we maximize the energy efficiency of a DD spectrum sharing, in IoT environment, where multiple LPUs and HPUs co-exist in the same area. We construct a multiobjective optimization problem to maximize the combined HP's and LP's EE performance. Analytical expressions are provided for the optimal variables under many scenarios. Numerical results have shown a large gain by jointly optimizing the spatial density and transmission power in compared to individual optimization, reaches up-to 2-4 order of magnitude. It also showed that the dominant system which decides the overall $\mathrm{EE}$ is the system corresponding to the lowest power for low/high power ratio.

\section{APPENDIX A ProOF OF PROPOSITION 1}

To proof Proposition 1, we must show that utilizing the generalized theory of convex optimization, i.e., Lagrangian function and KKT conditions, applies to problem (21). Hence, in here, we proof the strict quasi-concavity of $\mathcal{E}_{T S}$ in $P_{n}$ and $P_{n p}$. The necessity of proving the strictness is because in Alg. 1 we will utilize this property to propose an iterative global solution for problem 20. The proof of strict quasi-concavity of $\mathcal{E}_{T S}$ with respect to $P_{n}$ is easily derived, many work have done close proofs [4]. Whereas, the strict quasi-concavity of $\mathcal{E}_{T S}$ with respect to $P_{n p}$ is easily observed since the function is strictly decreasing on $P_{n p}$. To derive the expression in (23), we must observe that maximizing $\mathcal{E}_{T S}$ with respect to $P_{n}$ is equivalent to the following problem,

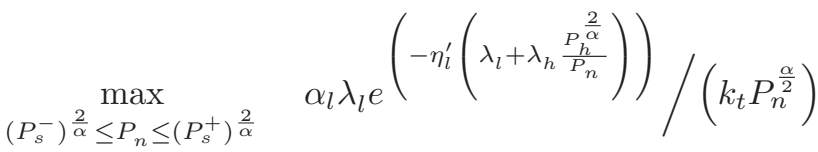

$$
\begin{aligned}
& \text { s.t. } \quad P_{n}=P_{n p}
\end{aligned}
$$



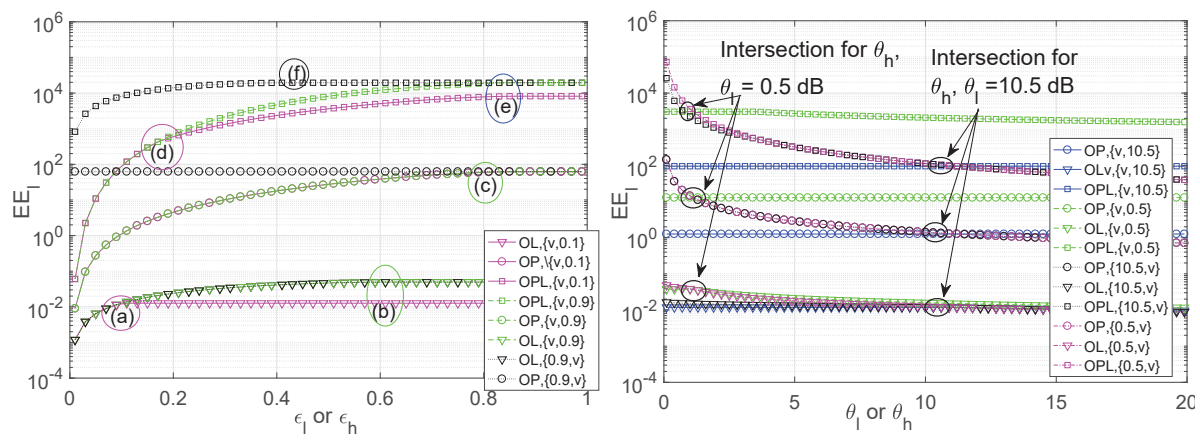

(a) LPS's EE versus tolerance threshold. Param- (b) LPS's EE versus SIR. Parameters: $P_{l,}=$ eters: $\quad P_{l, h}=25 \mathrm{dBm}, \theta_{l, h}=10.5 \mathrm{~dB}, R_{l, h}=2 \mathrm{~m}, k_{t}=1,25 \mathrm{dBm}, \epsilon_{l, h}=0.3, R_{l, h}=2 \mathrm{~m}, k_{t}=1, \lambda_{l, h}=0.5 \mathrm{e}-4$. $\lambda_{l, h}=0.5 \mathrm{e}-4$.

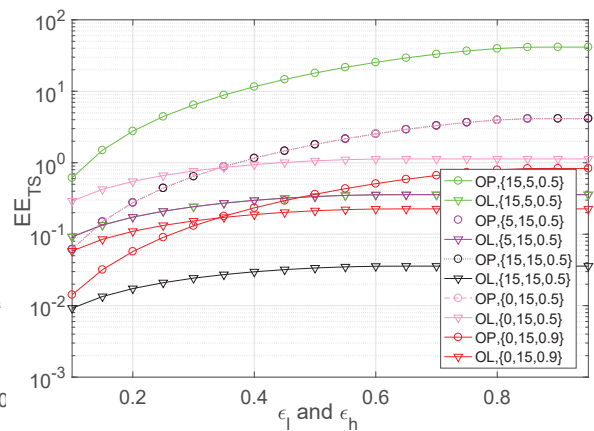

(c) Combined LPS and HPS EE versus tolerance threshold. Parameters: $R_{l, h}=4 \mathrm{~m}, \epsilon_{l, h}=$ $0.2, \theta_{l, h}=4 \mathrm{~dB}, k_{t}=1, \lambda_{l, h}=1 \mathrm{e}-4$.

Fig. 1. Overall Energy Efficiency Numerical Results

Note that the solution of this problem is different than that of (9) because we including the equality constraint $P_{n}=P_{n p}$. We then use the transformation on the objective function in (37a) using monotone increasing function, i.e., $\log ($.$) .$ The corresponding Lagrangian function of problem (37) is expressed as follows,

$\mathfrak{L}=\log \left(\frac{\alpha_{s} \lambda_{l}}{k_{t}}\right)-\eta_{s}^{\prime} \lambda_{l}-\eta_{s}^{\prime} \lambda_{h} \frac{P_{u}}{P_{n}}-\frac{\alpha}{2} \log \left(P_{n}\right)+\mu\left[P_{n}-P_{n p}\right]$,

where $P_{u}=P_{h}^{\frac{2}{\alpha}}$. Taking the derivative of the Lagrangian function in (38) and finding its zeros results in the following,

$$
2 \mu P_{n}^{2}-\alpha P_{n}+2 \eta_{s}^{\prime} \lambda_{h} P_{u}=0 .
$$

Hence, the expression in (23) is obtained by solving (39).

We then derive the expression in (25), by observing that maximizing $\mathcal{E}_{T S}$ with respect to $P_{n p}$ is equivalent to the following problem,

$$
\max _{\left(P_{s}^{-}\right)^{\frac{2}{\alpha}} \leq P_{n p} \leq\left(P_{s}^{+}\right)^{\frac{2}{\alpha}}} \alpha_{h} \lambda_{h} e^{\left(-\eta_{h}^{\prime}\left(\lambda_{h}+\frac{\lambda_{l} P_{n p}}{P_{h}^{\frac{2}{\alpha}}}\right)\right)} /\left(k_{t} P_{h}\right)
$$

s.t.

$$
P_{n}=P_{n p} .
$$

We then formulate the Lagrangian function of (40), devise its first derivative and find its zeros as follows,

$$
\frac{-\alpha_{p} \lambda_{h} \eta_{p}^{\prime} \lambda_{l} e^{-\eta_{p}^{\prime} \lambda_{h}}}{k_{t} P_{u}^{\frac{\alpha}{2}}} \exp \frac{-\eta_{p}^{\prime} \lambda_{l} P_{n p}}{P_{u}}+\mu=0
$$

Solving (41) leads to the exact solution in (25).

\section{APPENDIX B}

\section{Proof OF THEOREM 2}

To proof Theorem we need to satisfies the conditions of the findings in [14]. Grippo and Sciandrone, in [14], verified that using Gauss-Seidel method to optimize over several variable guarantee a global optimal solution without the necessity of proving joint concavity/convexity structure. The condition which must be met is to prove that the targeted problem is strict quasi-concave with respect to to each individual variable. Therefore, to proof $\mathrm{B}$, it is enough to verify the strict quasiconcave property of problem $\mathcal{E}_{T S}$ with respect to each variable
$P_{n}, P_{n p}, P_{u}, P_{u s}, \lambda_{l}, \lambda_{s p}$. However, note that we have already proven the strict quasi-concave of the problem with respect to $P_{n}, P_{n p}$, and $\lambda_{l}$ in the associated appendices. Similarly, we can prove the strict quasi-concave structure of $\mathcal{E}_{T S}$ with respect to $P_{u}, P_{u s}$, and $\lambda_{h}$. This concludes the proof of Theorem 2 .

\section{REFERENCES}

[1] W. Ye, J. Heidemann, and D. Estrin, "Medium access control with coordinated adaptive sleeping for wireless sensor networks," IEEE/ACM Transactions on Networking, vol. 12, no. 3, pp. 493-506, June 2004.

[2] A. Alabbasi, Z. Rezki, and B. Shihada, "Energy efficient resource allocation for cognitive radios: A generalized sensing analysis," IEEE Transactions on Wireless Communications, vol. 14, no. 5, pp. 24552469, May 2015.

[3] J. Wu, G. Wang, and Y. Zheng, "Energy efficiency and spectral efficiency tradeoff in Type-I ARQ systems," IEEE Journal on Selected Areas in Communications, vol. 32, no. 2, pp. 356-366, February 2014.

[4] A. Alabbasi and B. Shihada, "Optimal cross-layer design for energy efficient d2d sharing systems," IEEE Transactions on Wireless Communications, vol. 16, no. 2, pp. 839-855, Feb 2017.

[5] M. Haenggi, Stochastic Geometry for Wireless Networks. Cambridge University Press, 2012.

[6] T. Q. S. Quek, W. C. Cheung, and M. Kountouris, "Energy efficiency analysis of two-tier heterogeneous networks," in Wireless Conference 2011 - Sustainable Wireless Technologies (European Wireless), 11th European, April 2011, pp. 1-5.

[7] Y. Kwon and T. Hwang, "A game-theoretic approach for energyefficient power control in spectrum sharing networks," in 2014 IEEE International Conference on Communications (ICC), June 2014, pp. 3493-3498.

[8] R. Hernandez-Aquino, S. A. R. Zaidi, D. McLernon, and M. Ghogho, "Energy efficiency analysis of two-tier mimo diversity schemes in poisson cellular networks," IEEE Transactions on Communications, vol. 63, no. 10, pp. 3898-3911, Oct 2015.

[9] H. H. Yang, G. Geraci, and T. Q. S. Quek, "Energy-efficient design of mimo heterogeneous networks with wireless backhaul," IEEE Transactions on Wireless Communications, vol. PP, no. 99, pp. 1-1, 2016.

[10] W. Nie, Z. Yi, F. Zheng, W. Zhang, and T. O'Farrell, "Hetnets with random dtx scheme: Local delay and energy efficiency," IEEE Transactions on Vehicular Technology, vol. PP, no. 99, pp. 1-1, 2015.

[11] M. Haenggi and R. K. Ganti, "Interference in large wireless networks," Foundations and Trends in Networking, vol. 3, no. 2, pp. 127-248, 2008. [Online]. Available: http://dx.doi.org/10.1561/1300000015

[12] S. Boyd and L. Vandenberghe, Convex Optimization, 2004.

[13] A. Alabbasi. (2017) On Energy Efficiency of Prioritiezed IoT System: KTH Technical Report. www.shihada.com/node/publications/EE_PIoT_ Long.pdf.

[14] L. Grippo and M. Sciandrone, "On the convergence of the block nonlinear Gauss-Seidel method under convex constraints," Operations Research Letters, vol. 26, no. 3, pp. 127 - 136, 2000. 